\title{
Study protocol: high-dose mizoribine with prednisolone therapy in short-term relapsing steroid-sensitive nephrotic syndrome to prevent frequent relapse (JSKDC05 trial)
}

Taketsugu Hama ${ }^{1}$, Koichi Nakanishi ${ }^{*}$ (D, Kenji Ishikura ${ }^{3}$, Shuichi Ito ${ }^{4}$, Hidefumi Nakamura ${ }^{5}$, Mayumi Sako ${ }^{6}$, Mari Saito-Oba ${ }^{7}$, Kandai Nozu ${ }^{8}$, Yuko Shima ${ }^{1}$, Kazumoto lijima ${ }^{8}$, Norishige Yoshikawa ${ }^{9}$ and for the Japanese Study Group of Kidney Disease in Children (JSKDC)

\begin{abstract}
Background: Eighty percent of children with steroid-sensitive nephrotic syndrome (SSNS) relapse within 2 years and 40-50\% patients show frequently-relapsing nephrotic syndrome (FRNS). Patients showing a relapse within 6 months after initial remission are at high risk of FRNS. Since frequent prednisolone treatment for FRNS induces severe prednisolone side effects, development of a treatment to prevent patients from shifting to FRNS is desirable. Mizoribine is an immunosuppressive drug with fewer side effects than prednisolone. Recent studies reported the efficacy of high-dose mizoribine in children with FRNS.

Methods/design: We conduct a multicenter, open, randomized controlled trial to investigate the efficacy and safety of standard prednisolone plus high-dose mizoribine therapy in children with SSNS showing a relapse within 6 months after an initial remission. Patients are allocated to either standard prednisolone alone treatment group, or standard prednisolone plus high-dose mizoribine group. For the former group, mizoribine is administered at a dose of $10 \mathrm{mg} / \mathrm{kg} /$ day once daily and continued for 2 years. The primary endpoint is the duration to frequent relapse.

Discussion: The results provide important data on use of high-dose mizoribine to prevent SSNS patients from shifting to FRNS. Since blood concentrations of mizoribine have not been investigated in detail until now, there is a possibility that mizoribine is underestimated in favor of other immunosuppressive drugs. In future, high-dose mizoribine therapy may lead to prevention of relapse in children at high risk of FRNS, and to decreased total dose of prednisolone.
\end{abstract}

Trial registration: UMIN000005103, (Prospectively registered 1st March 2011).

Keywords: Mizoribine, Frequently-relapsing nephrotic syndrome, Steroid-sensitive nephrotic syndrome

\footnotetext{
* Correspondence: knakanis@med.u-ryukyu.ac.jp

${ }^{2}$ Department of Child Health and Welfare (Pediatrics), Graduate School of

Medicine, University of the Ryukyus, 207 Uehara, Nishihara, Okinawa

903-0215, Japan

Full list of author information is available at the end of the article
}

(c) The Author(s). 2018 Open Access This article is distributed under the terms of the Creative Commons Attribution 4.0 International License (http://creativecommons.org/licenses/by/4.0/), which permits unrestricted use, distribution, and reproduction in any medium, provided you give appropriate credit to the original author(s) and the source, provide a link to the Creative Commons license, and indicate if changes were made. The Creative Commons Public Domain Dedication waiver (http://creativecommons.org/publicdomain/zero/1.0/) applies to the data made available in this article, unless otherwise stated. 


\section{Background}

Prednisolone (PSL) is the first-line drug for the treatment of idiopathic nephrotic syndrome (NS) in children. Ninety percent of patients with childhood NS have steroid-sensitive nephrotic syndrome (SSNS) and remit within 4 weeks after initiation of PSL therapy. After remission, patients require no drug treatment until relapse. Approximately $80 \%$ of patients with SSNS relapse within 2 years of PSL treatment as part of the International Study of Kidney Disease in Children (ISKDC) regimen. Around 40 to $50 \%$ of patients shift to either "frequently-relapsing NS (FRNS)" as repeating relapse in a relatively short period, or "steroid-dependent NS (SDNS)" as relapsing with decreasing or stopping PSL [1].

Frequent PSL treatment becomes problematic because of the side effects of steroids in FRNS patients. It is clear that patients who relapse immediately (within 6 months) after remission by PSL treatment for the first episode of NS tend to shift to FRNS [2]. It is therefore desirable to inhibit patients from relapsing soon after remission and to avoid the shift to FRNS.

Mizoribine is known to be safer than other immunosuppressive drugs due to its pharmacological characteristics [3, 4]. A placebo-controlled randomized trial in children with FRNS demonstrated that mizoribine has an inhibitory effect for relapsing only in patients 10 years old or younger [3]. Conventional use of mizoribine (3-4 mg/kg daily) leads to a peak blood level at around $1.0 \mu \mathrm{g} / \mathrm{mL}$ [5] although a peak blood level of $2.5-3.0 \mu \mathrm{g} / \mathrm{mL}$ is needed to sustain long-term efficacy for treatment of glomerular diseases [6, 7]. It is thought that children need to take double the dosage of mizoribine as adults because the volume of extracellular fluid of children is larger than in adults and interstitial drug absorption efficacy of children might be lower [8-11].

Recently, high-dose mizoribine administrations aimed to keep high serum mizoribine concentration have been considered for children with FRNS $[12,13]$. Serum concentration after $2 \mathrm{~h}$ of mizoribine administration (C2) was about $3 \mu \mathrm{g} / \mathrm{mL}$ in patients who achieved decrease in the number of relapses. These patients took $10 \mathrm{mg} / \mathrm{kg} /$ day of mizoribine once a day, and there were no severe side effects.

Patients with NS under 10 years old and who relapse within 6 months after remission for the first episode of NS are considered to be high risk of FRSN [2]. We therefore focus on the possibility that adding high-dose mizoribine to the standard PSL treatment for SSNS patients who are likely to shift to FRNS could prevent them from shifting to FRNS. Here, we conduct a multicenter open randomized controlled trial to evaluate the efficacy and safety of PSL plus high-dose mizoribine treatment.

\section{Methods/design}

Aim

This trial aims to investigate whether standard PSL plus high-dose mizoribine combination therapy is superior to standard PSL alone in preventing a shift to FRNS for children with SSNS who immediately (within 6 months) relapse after remission with the ISKDC PSL treatment for the first episode of NS.

\section{Study design and patients}

We conduct a multicenter, randomized, superiority, open-label trial and compare standard PSL plus high-dose mizoribine combination treatment with standard PSL alone treatment for children with SSNS who immediately (within 6 months) relapse after remission for the first episode of NS (Fig. 1). We diagnose NS and remission according to the ISKDC. Patients aged 2-10 years are eligible if they have remission within

3 weeks of PSL administration for the first relapse.

Inclusion and exclusion criteria are as follows:

Inclusion criteria:

1. Patients diagnosed with a first episode of NS who meet (a) urinary protein/creatinine ratio $\geq 1.8$ and (b) $\leq 2.5 \mathrm{~g} / \mathrm{dL}$ of serum albumin

2. Patients treated by the ISKDC based regimen at the first episode and relapsed within 6 months after the first remission

3. Age at the registration $\geq 2$ and $<11$ years

4. Patients who remit within 3 weeks after PSL treatment for the first relapse

Exclusion criteria:

1. Patients diagnosed with nephritic NS

2. Patients diagnosed with IgA vasculitis, systemic lupus nephritis or secondary NS

3. Patients who have been diagnosed with steroidresistant NS (SRNS)

4. Patients who have been treated with any immunosuppressant for NS

5. Patients with poorly controlled hypertension, impaired renal function, or severe liver dysfunction

6. Patients who are participating in other studies at the time of registration.

\section{Randomization}

Patients are randomly assigned to either the standard PSL plus high-dose mizoribine combination, or standard PSL alone group in a 1:1 ratio. We use a minimization method stratifying by age (2-5 years or 6-10 years), sex, duration from initial remission to relapse ( $<90$ days or $\geq$ 90 days) and institution as adjustment (stratification) factors. Patients, their guardians, treating physicians, and individuals assessing outcomes and analyzing data are 


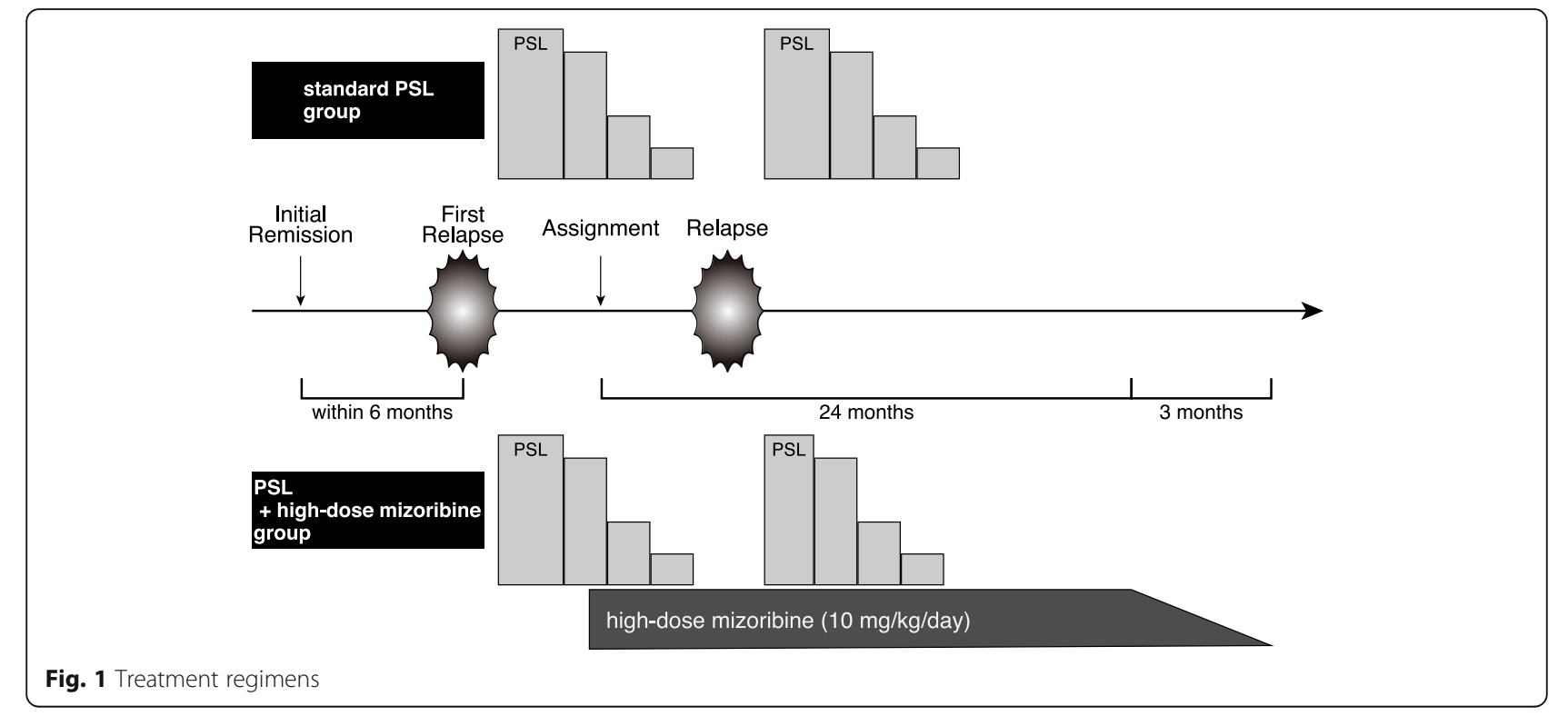

not blinded to the patients' treatment assignments. Apart from the trial statistician and the data-monitoring committee, all treating physicians and other investigators remain blinded to the trial results until follow-ups are completed.

\section{Procedures}

For the standard PSL plus high-dose mizoribine group, mizoribine is administered at a dose of $10 \mathrm{mg} / \mathrm{kg} /$ day (maximum $300 \mathrm{mg} /$ day) once daily after breakfast and continued for 2 years. Mizoribin is started within 7 days from the registration date. After 2-year administration or termination of this trial, dosage of mizoribine will be tapered every month and stopped within 3 months. In case of leukocytopenia or impaired renal function, mizoribine treatment will be tapered or stopped. PSL treatment for relapse during the observation period follows the ISKDC regimen.

The observation period is from the registration date to the completion or discontinuation date of this trial. Completion of study is defined as completing of 2-year administration. Patients who complete or discontinue this study will be followed-up.

\section{Outcomes}

The primary endpoint is defined as the duration from registration to diagnosis of FRNS. Diagnosis of FRNS is based on relapse dates according to the ISKDC. In our study, FRNS is defined as two relapses within 6 months of initial remission, or four relapses within any 12month period. This includes relapses during initial tapering treatment, but excludes relapses with spontaneous remissions. Relapse is defined as proteinuria $2+$ or higher on dipstick analysis for 3 or more consecutive days or proteinuria $2+$ or higher on dipstick analysis and serum albumin $\leq 2.5 \mathrm{~g} / \mathrm{dL}$. Immunosuppressant administration is prohibited in the protocol. If patients show SDNS or SRNS, they are treated as an event in the primary analysis. Data for patients who do not experience these events are considered censored at the last examination. Secondary endpoints are relapse-free period, the poportion of patients without relapses, the number of relapses per person/year, time to SDNS, time to SRNS, total prednisolone dose, and adverse events. Adverse events were recorded throughout the trial period and assessed using Common Terminology Criteria for Adverse Events v3.0. Serum mizoribine concentrations at trough and C3 are measured 1 month after administration of mizoribine to monitor safety and pharmacological effect.

\section{Statistical analyses}

The primary analysis of this study is to examine the superiority of PSL plus high-dose mizoribine combination therapy compared to standard PSL alone therapy in the time to FRNS.

For the sample size determination, we based the hazard rate ratio (HR, the PSL plus high dose mizoribine treatment group to the PSL treatment group) on the two-year FRNS probability. We estimated 50\% at two-year in the standard PSL treatment and postulated the $\mathrm{HR}$ of 0.50 . The required sample size was calculated as 108 patients (54 per arm) under a two-sided alpha $5 \%$ and $80 \%$ power, if the patients accrued for 4 years and follow up for 2 years. Considering withdrawal of consent or loss, target sample number was determined as 120 cases (60 each). We used POWER procedure of SAS software (version 9.3) for this calculation. 
The primary endpoint, time to FRNS will be compared between these two groups using log-rank test. Cox proportional hazard model is used to estimate the hazard ratio with a $95 \%$ confidence interval. Kaplan-Meier method is used to summarize time to FRNS. The statistical significance level is set to $5 \%$.

Secondary endpoints of relapse-free period and duration shifting to SDNS or SRNS will be compared in a similar manner as primary endpoint. The proportion of patients without relapses will be compared by Fisher's exact test. Number of relapse and total steroid dose will be analyzed by Wilcoxson test. Correlation between mizoribine dose and blood concentration of mizoribine will be evaluated by Spearman rank correlation coefficient analysis.

\section{Discussion}

The purpose of this trial is to examine efficacy and safety of high-dose mizoribine in preventing patients with NS who show an early relapse within 6 months after initial remission from shifting to FRNS. One of most noteworthy adverse events in patients with SSNS is harmfulness of steroids, especially to those with FRNS/SDNS. It has been clarified that prolonged ISKDC steroid regimen for patients with SSNS did not shorten the relapse-free period $[2,14-16]$. It is therefore reasonable to pursue new strategies to prevent patients with SSNS from shifting to FRNS. Although immunosuppressants are considered as second-step drugs next to steroids, use of them should be carefully decided due to severe side effects. Under such a condition, it is important to recognize predictive risk factors for FRNS. Previously, we clarified that a relapse within 6 months after initial treatment for NS is a predictive risk factor for FRNS [2]. Development of an optional drug regimen for patients who have such a risk factor is therefore desirable. From this perspective, we focused on mizoribine and conducted a randomized controlled trial to examine efficacy and safety of adding optional mizoribine to patients with NS.

Mizoribine has been developed in Japan. It is an imidazole-based nucleic acid-related substance derived from the culture fluid of Eupenicillium brefeldianum. Mizoribine is an antimetabolite to inhibit the purine synthesis pathway of nucleic acids, it shows an immunosuppressive effect of more selectively inhibiting lymphocyte proliferation [17, 18]. Unlike other immunosuppressive drugs, such as cyclosporine and cyclophosphamide, toxicity and myelosuppression of mizoribine seems to be milder. Mizoribine is also considered to have an effect to enhance glucocorticoid efficacy [19].

Historically, mizoribine has been used to suppress rejection reactions in kidney transplantation [20,21], lupus nephritis [22, 23] and rheumatoid arthritis. Mizoribine has also been reported to be effective as a treatment for IgA nephropathy $[24,25]$. It is approved as a treatment of NS in Japan $[3,26]$. By adding mizoribine, the total dose of steroids can reportedly be reduced in treatment for NS. [27]. Furthermore, a double-blind, placebo-controlled, multicenter trial has shown that mizoribine significantly decreased relapse rate and prolonged remission period in children with FRNS in the subgroup consisting of patients 10 years old or younger [3]. Although efficacy and safety of mizoribine are well recognized in medical practice, blood concentration of mizoribine has not always been sufficient to reach efficacy. There is therefore a possibility that mizoribine is underestimated compared to other immunosuppressive drugs. Conventional use of mizoribine $(3-4 \mathrm{mg} / \mathrm{kg}$ daily, maximum $150 \mathrm{mg} /$ day) leads to a peak blood level at around $1.0 \mu \mathrm{g} / \mathrm{mL}$ [5], although a peak blood level of $2.5-3.0 \mu \mathrm{g} / \mathrm{mL}$ is biologically required to sustain long-term efficacy for the treatment of glomerular diseases $[6,7]$. Consequently, high-dose mizoribine administrations aiming to retain high serum mizoribine concentration have come to be considered for children with FRNS $[12,13]$. Serum C2 concentrations are known to be around $3 \mu \mathrm{g} / \mathrm{mL}$ in patients who achieved decrease in the number of relapses by taking $10 \mathrm{mg} / \mathrm{kg} /$ day of mizoribine once a day without severe side effects. Therefore, in the current protocol, we adopted dosage of $10 \mathrm{mg} / \mathrm{kg} /$ day of mizoribine once a day (maximum $300 \mathrm{mg} /$ day).

If we can prevent at-risk patients from shifting to FRNS by adding high-dose mizoribine effectively and safely, we will reduce the total dose of steroids for patients, resulting in less side effects. Moreover, we will avoid use of other immunosuppressants, such as cyclosporine and cyclophosphamide, which are proven to be more effective but have more serious side effects than mizoribine. Patients with mizoribine treatment do not require frequent blood tests or renal biopsy, which is seen as a great benefit. As a result, it is expected to reduce medical expenses despite cost of mizoribine.

\section{Summary}

The present study aims to examine efficacy and safety of standard PSL plus high-dose mizoribine therapy used to prevent at-risk children with SSNS from shifting to FRNS. An early relapse within 6 months after initial remission in patients with SSNS is a definitive risk for the shift to FRNS. High-dose mizoribine administration promises biological and clinical bases for efficacy. These conditions give the protocol strong rationales. The results of this trial may be of great benefit to children with SSNS at risk of FRNS and to their families.

\section{Abbreviations}

FRNS: frequently relapsing nephrotic syndrome; ISKDC: International Study of Kidney Disease in Children; NS: nephrotic syndrome; PSL: prednisolone;

SSNS: steroid sensitive nephrotic syndrome 


\section{Acknowledgements}

The authors are grateful to the participating JSKDC05 trial team for their precious work.

\section{Funding}

The trial is supported by a grant from the Ministry of Health, Labor and Welfare, Japan. Mizoribine is provided free of charge by Asahi Kasei Pharma Corporation. The sponsor approved the study protocol, but had no role in data collection, data analysis and drafting or approving the present manuscript.

\section{Availability of data and materials}

Not applicable for this publication.

\section{Authors' contributions}

All authors have contributed to study design and preparation of this manuscript. KON is the PI for the JSKDC05 study. KON, Kl, SI, HN and MS leads on study design and analytical plan. MSO is the statistician responsible for all study procedures. KON, YS and KAN leads on research governance. KI and NY are Chief Investigators with overall responsibility for JSKDC studies. TH and KON wrote the first draft of this manuscript; all co-authors critically reviewed and revised the initial draft and approved the final version of the manuscript.

\section{Ethics approval and consent to participate}

This study is performed in accordance with the Declaration of Helsinki and is approved by the regional research ethics vetting boards (Wakayama Medical University). Before enrollment, patients' guardians provided written informed consent, and informed assent was obtained from older children.

\section{Consent for publication}

\section{Not applicable.}

\section{Competing interests}

KON received a lecture fee from Asahi Kasei Pharma Corp. KI received grants from Asahi Kasei Pharma Corp. and Pfizer Japan Inc., and a lecture fee from Asahi Kasei Pharma Corp. SI received grants from Asahi Kasei Pharma Corp. and Pfizer Japan Inc., and a lecture fee from Asahi Kasei Pharma Corp, Pfizer Japan Inc. and Shionogi \& Co., Ltd. HN is a stockholder of Asahi Kasei Pharma Corp. KAN received a lecture fee from Asahi Kasei Pharma Corp. Kl has received a grant from Takeda Pharmaceutical Co., Ltd., and lecture fees and/or consulting fees from Asahi Kasei Pharma Corp. and Takeda Pharmaceutical Co., Ltd. The other authors had no disclosure to declare.

\section{Publisher's Note}

Springer Nature remains neutral with regard to jurisdictional claims in published maps and institutional affiliations.

\footnotetext{
Author details

'Department of Pediatrics, Wakayama Medical University, Wakayama, Japan. ${ }^{2}$ Department of Child Health and Welfare (Pediatrics), Graduate School of Medicine, University of the Ryukyus, 207 Uehara, Nishihara, Okinawa 903-0215, Japan. ${ }^{3}$ Division of Nephrology and Rheumatology, National Center for Child Health and Development, Setagaya-ku, Tokyo, Japan. ${ }^{4}$ Department of Pediatrics, Yokohama City University, Yokohama, Kanagawa, Japan. ${ }^{5}$ Clinical Research Center, National Center for Child Health and Development, Setagaya-ku, Tokyo, Japan. ${ }^{6}$ Division of Clinical Trials, Department of Clinical Research Promotion, Clinical Research Center, National Center for Child Health and Development, Setagaya-ku, Tokyo, Japan. 'Department of Medical Statistics, Toho University School of Medicine, Ota-ku, Tokyo, Japan. ${ }^{8}$ Department of Pediatrics, Kobe University Graduate School of Medicine, Kobe, Hyogo, Japan. ${ }^{9}$ Clinical Research Center, Wakayama Medical University, Wakayama, Japan.
}

Received: 21 June 2018 Accepted: 2 September 2018 Published online: 10 September 2018

\section{References}

1. Tarshish P, Tobin JN, Bernstein J, Edelmann CM. Prognostic significance of the early course of minimal change nephrotic syndrome: report of the international study of kidney disease in children. J Am Soc Nephrol. 1997:8:769-76.
2. Nakanishi K, lijima K, Ishikura K, Hataya H, Nakazato H, Sasaki S, et al. Twoyear outcome of the ISKDC regimen and frequent-relapsing risk in children with idiopathic nephrotic syndrome. Clin J Am Soc Nephrol. 2013;8:756-62.

3. Yoshioka K, Ohashi Y, Sakai T, Ito H, Yoshikawa N, Nakamura H, et al. A multicenter trial of mizoribine compared with placebo in children with frequently relapsing nephrotic syndrome. Kidney Int. 2000;58:317-24.

4. Kuroda T, Hirose S, Tanabe N, Sato H, Nakatsue T, Ajiro J, et al. Mizoribine therapy for patients with lupus nephritis: the association between peak mizoribine concentration and clinical efficacy. Mod Rheumatol. 2007;17:206-12.

5. Tanaka H, Suzuki K, Nakahata T, Tsugawa K, Ito E, Waga S. Mizoribine oral pulse therapy for patients with disease flare of lupus nephritis. Clin Nephrol. 2003;60:390-4.

6. Tanaka H, Tsugawa K, Suzuki K, Nakahata T, Ito E. Long-term mizoribine intermittent pulse therapy for young patients with flare of lupus nephritis. Pediatr Nephrol. 2006;21:962-6.

7. Tanaka H, Oki E, Tsuruga K, Sato N, Matsukura H, Matsunaga A, et al. Mizoribine treatment of young patients with severe lupus nephritis: a clinicopathologic study by the tohoku pediatric study group. Nephron Clin Pract. 2008;110:c73-9.

8. Fuke T, Abe Y, Mikawa T, Oto H, Iwasaki J, Tsuji Y, et al. Pharmacokinetics and Administration Planning of Mizoribine in Pediatric-onset Renal Disease Patients. [In Japanese only] Nihon Shoni Jinzobyo Gakkai Zasshi. 2008;21:10-16.

9. Nagai T, Uemura O, Kaneda H, Ushijima K, Ohta K, Gotoh Y, et al. The true distribution volume and bioavailability of mizoribine in children with chronic kidney disease. Clin Exp Nephrol. 2017;21:884-8.

10. Kaneda H, Shimizu M, Ohta K, Ushijima K, Gotoh Y, Satomura K, et al. Population pharmacokinetics of mizoribine in pediatric patients with kidney disease. Clin Exp Nephrol. 2016;20:757-63.

11. Goto M, Ikeda M, Hataya H, Ishikura K, Hamasaki Y, Honda M. Beneficial and adverse effects of high-dosage MZR therapy in the management of children with frequently relapsing nephrotic syndrome. [in Japanese only]. Nihon Jinzo Gakkai Shi. 2006:48:365-70.

12. Ohtomo Y, Fujinaga S, Takada M, Murakami H, Akashi S, Shimizu T, et al. High-dose mizoribine therapy for childhood-onset frequently relapsing steroid-dependent nephrotic syndrome with cyclosporin nephrotoxicity. Pediatr Nephrol. 2005;20:1744-9.

13. Kawasaki Y, Takano K, Isome M, Suzuki J, Suyama K, Kanno H, et al. Efficacy of single dose of oral mizoribine pulse therapy two times per week for frequently relapsing nephrotic syndrome. J Nephrol. 2007;20:52-6.

14. Yoshikawa N, Nakanishi K, Sako M, Oba MS, Mori R, Ota E, et al. A multicenter randomized trial indicates initial prednisolone treatment for childhood nephrotic syndrome for two months is not inferior to six-month treatment. Kidney Int. 2015;87:225-32.

15. Teeninga N, Kist-van Holthe JE, van Rijswijk N, de Mos NI, Hop WCJ, Wetzels JFM, et al. Extending prednisolone treatment does not reduce relapses in childhood nephrotic syndrome. J Am Soc Nephrol. 2013;24:149-59.

16. Sinha A, Saha A, Kumar M, Sharma S, Afzal K, Mehta A, et al. Extending initial prednisolone treatment in a randomized control trial from 3 to 6 months did not significantly influence the course of illness in children with steroid-sensitive nephrotic syndrome. Kidney Int. 2015;87:217-24.

17. Yokota S. Mizoribine: mode of action and effects in clinical use. Pediatr Int. 2002:44:196-8.

18. Kawasaki Y. Mizoribine: a new approach in the treatment of renal disease. Clin Dev Immunol. 2009;2009:681482.

19. Takahashi S, Wakui H, Gustafsson JA, Zilliacus J, Itoh H. Functional interaction of the immunosuppressant mizoribine with the 14-3-3 protein. Biochem Biophys Res Commun. 2000;274:87-92.

20. Uchida H, Mita K, Bekku Y, Nishimura Y, Ishida Y, Watanabe K, et al. Advantages of triple therapy with mizoribine, cyclosporine and prednisolone over other types of triple and/or double therapy including cyclosporine for renal transplantation. J Toxicol Sci. 1991;16:181-90.

21. Sonda K, Takahashi K, Tanabe K, Funchinoue S, Hayasaka Y, Kawaguchi H, et al. Clinical pharmacokinetic study of mizoribine in renal transplantation patients. Transplant Proc. 1996;28:3643-8.

22. Tanaka H, Tsugawa K, Tsuruga K, Suzuki K, Nakahata T, Ito E, et al. Mizoribine for the treatment of lupus nephritis in children and adolescents. Clin Nephrol. 2004;62:412-7.

23. Yumura W, Suganuma S, Uchida K, Moriyama T, Otsubo S, Takei T, et al. Effects of long-term treatment with mizoribine in patients with proliferative lupus nephritis. Clin Nephrol. 2005;64:28-34. 
24. Kaneko T, Hirama A, Ueda K, Fujino T, Utsumi K, lino Y, et al.

Methylprednisolone pulse therapy combined with mizoribine following

tonsillectomy for immunoglobulin a nephropathy: clinical remission rate,

steroid sparing effect, and maintenance of renal function. Clin Exp Nephrol. 2011;15:73-8.

25. Yoshikawa N, Nakanishi K, Ishikura K, Hataya H, lijima K, Honda M, et al.

Combination therapy with mizoribine for severe childhood IgA

nephropathy: a pilot study. Pediatr Nephrol. 2008;23:757-63.

26. Honda M. Nephrotic syndrome and mizoribine in children. Pediatr Int 2002;44:210-6.

27. Igarashi Y, Moro Y, Kondo Y, Inoue CN. Steroid-sparing effect of mizoribine in long-term nephrotic syndrome of children. Pediatr Nephrol. 1994;8:396-7.

Ready to submit your research? Choose BMC and benefit from:

- fast, convenient online submission

- thorough peer review by experienced researchers in your field

- rapid publication on acceptance

- support for research data, including large and complex data types

- gold Open Access which fosters wider collaboration and increased citations

- maximum visibility for your research: over $100 \mathrm{M}$ website views per year

At BMC, research is always in progress.

Learn more biomedcentral.com/submissions 